\title{
A Generalized Approach Obeying the Third Law of Thermodynamics for the Expression of Lattice Stability and Compound Energy: A Case Study of Unary Aluminum
}

\author{
Toshihiro Omori $^{1,2} \cdot$ Sedigheh Bigdeli $^{1} \cdot$ Huahai Mao ${ }^{1,3}$
}

Submitted: 15 May 2018/in revised form: 29 May 2018/Published online: 11 June 2018

(c) The Author(s) 2018

\begin{abstract}
Recently, Hillert and Selleby proposed a simple method for expression of the lattice stability or Gibbs energy of formation that does not violate the third law of thermodynamics. This method describes the derivation of the Gibbs energy function from high temperatures down to $0 \mathrm{~K}$ by interpolation, instead of extrapolation from room temperature to $0 \mathrm{~K}$. In the present work, their original method is discussed in terms of determination of the characteristic parameter values. Keeping the essential interpolation character of their method, a generalized approach is presented for expressing the lattice stability through parameter optimizations. This approach retains the zero point entropy of substances and is in line with the development of the third generation CALPHAD databases. Using the Al unary system as a case study, the lattice stabilities of the hcp and bcc phases are investigated. The respective Einstein temperatures are also evaluated. At high temperatures, the present descriptions reproduce the lattice stabilities suggested by SGTE for the existing second generation of databases, with a reasonable accuracy.
\end{abstract}

This invited article is part of a special issue of the Journal of Phase Equilibria and Diffusion in honor of Prof. Zhanpeng Jin's 80th birthday. The special issue was organized by Prof. Ji-Cheng (JC) Zhao, The Ohio State University; Dr. Qing Chen, Thermo-Calc Software AB; and Prof. Yong Du, Central South University.

Toshihiro Omori

tomori@kth.se; omori@material.tohoku.ac.jp

1 Department of Materials Science and Engineering, KTH Royal Institute of Technology, 10044 Stockholm, Sweden

2 Department of Materials Science, Graduate School of Engineering, Tohoku University, Sendai 980-8579, Japan

3 Thermo-Calc Software AB, Råsundavägen 18, 16967 Solna, Sweden
More importantly, information from ab initio calculations (total energy at $0 \mathrm{~K}$ ) is also used for this optimization and the present method results in a physically sounder description of thermodynamic properties at lower temperatures down to $0 \mathrm{~K}$. The present approach provides a simple and flexible way to estimate the lattice stabilities, with potential applicability for the Gibbs energy of formation of stoichiometric compounds and the excess energy of solution phases, in accordance with the third law of thermodynamics.

Keywords aluminum $\cdot$ excess energy $\cdot$ Gibbs energy of formation - lattice stability - thermodynamic database . third generation of CALPHAD databases

\section{Introduction}

In the CALculation of PHAse Diagrams (CALPHAD) method, the Gibbs energy is described as a function of temperature, pressure and content of components. The parameters of the Gibbs energy function based on some model with a mathematical expression are determined so as to represent the information of thermodynamic quantities and phase diagram. The description of the pure elements by Scientific Group Thermodata Europe (SGTE) using the empirical polynomial functions ${ }^{[1]}$ has widely been accepted, although it is restricted at temperatures mainly down to $298.15 \mathrm{~K}$ and the main application is in the high-temperature region. Until now, a wide range of thermodynamic databases has been developed, based on these unary descriptions, and are known as the second generation of thermodynamic databases.

Conversely, the third generation of CALPHAD databases with more physical basis is being developed, aiming 
for the thermodynamic descriptions reliable from $0 \mathrm{~K}$ up to high temperatures above the melting temperature. The ideas of the thermodynamic models considering each physical effect, including the Debye or Einstein model, were proposed at the Ringberg workshop, ${ }^{[2-4]}$ and later some attempts were made to develop an appropriate model. ${ }^{[5-8]}$ In the case of unary Fe studied by Chen and Sundman, ${ }^{[5]}$ the specific heat was described with help from the model shown later as $\mathrm{Eq} 7$, i.e., the harmonic lattice vibration described by the Einstein model, electronic excitation, anharmonic lattice vibration and magnetic contribution were modeled separately. More efforts have been made to assess the thermodynamic parameters based on this model using experimental information including specific heat and enthalpy in unaries $\mathrm{Fe},{ }^{[5,9]} \mathrm{Mn}^{[10]}$ and $\mathrm{Co}{ }^{[11]}$

One of the challenges in developing the thermodynamic database is to reasonably estimate the lattice stabilities of metastable and unstable phases, i.e., the differences in Gibbs energy between stable and metastable (unstable) phases for an element. The pressure dependence of Gibbs energy is not discussed in this paper. The temperature dependence of the Gibbs energy of a metastable (unstable) $\beta$ phase, relative to that of a stable $\alpha$ phase is often described using the following approximation as a linear expression for many pure elements in the SGTE database. This simple approach has been adopted due to the lack of experimental information ${ }^{[1,12]}$ :

$$
{ }^{\circ} G_{i}^{\beta}-{ }^{\circ} G_{i}^{\alpha}=a-b T
$$

This expression indicates that both phases have the same specific heat and that the difference in entropy of the $\beta$ phase against the $\alpha$ phase is $b$, independent of temperature. Therefore, the entropy of the $\beta$ phase extrapolated to $0 \mathrm{~K}$ is not zero, which is in disagreement with the third law of thermodynamics. The situation is often similar for the Gibbs energy of formation in compounds and excess energy of solution phases. Alternatively, one simple way to extend the Gibbs energy function and their derivations to $0 \mathrm{~K}$ by interpolation instead of extrapolation has been recently proposed by Hillert and Selleby ${ }^{[13]}$ as Eq 2:

$$
{ }^{\circ} G_{i}^{\beta}-{ }^{\circ} G_{i}^{\alpha}=a-b\left\{T+\frac{T_{1}}{n}\left(1-\frac{T}{T_{1}}\right)^{n}\right\} .
$$

During the last decade, the methodology of the theoretical calculations and computer technique development have made the ab initio calculations increasingly applied as a powerful tool for calculating the thermodynamic properties of materials, particularly for phases that cannot be experimentally examined. For example, in the case of $\mathrm{Al}$, the stable fcc phase has been studied by both experiments and theories. ${ }^{[14-21]}$ It was extensively discussed in terms of the individual contributions to the heat capacity (or entropy). ${ }^{[7,22-24]}$ The largest contribution is, of course, the harmonic phonon which can be described by Debye or Einstein models. The anharmonic effects are also important despite its smaller amount of contributions. One of them is the phonon frequencies shift due to the thermal expansion, often described by the quasiharmonic model. This effect occupies a dominant part of heat capacity beyond the harmonic contribution. The other is referred as the explicitly (or high-order) anharmonic effect. This contribution is negative in the fcc $\mathrm{Al}$, which is not a general result in substances, and partially compensates the electronic excitations. ${ }^{[23,24]}$ The vacancy contribution is small. $^{[24]}$ The hcp and bcc phases are metastable and unstable at $0 \mathrm{~K}$ under ambient pressure in $\mathrm{Al}$, respectively. ${ }^{[25-28]}$ The stability of these phases has been calculated and the effect of pressure has been a main issue because these structures become stable at high pressures. ${ }^{[26-42]}$ From a practical viewpoint of CALPHAD, the Gibbs energy has to be defined/estimated even for unstable phases at ambient pressure (such as bcc $\mathrm{Al}$ ), which determines the applicability and quality of the extrapolation of the CALPHAD data into high order systems (e.g. Al solubility in other metals with bcc structure) or higher pressures (of pure $\mathrm{Al}$ ). Moreover, reported experimental information of thermodynamic quantities at finite temperatures tends to be limited compared to that from groundstate calculations; nevertheless, it is necessary to assess the lattice stability for the application of CALPHAD data in multicomponent systems. One effective way to assess the lattice stability valid from $0 \mathrm{~K}$ up to high temperatures beyond liquidus is to couple the ab initio calculations with the existing SGTE descriptions into a new thermodynamic model implemented in the third generation CALPHAD database. The above mentioned Eq 2 is helpful to describe the lattice stability in agreement with the third law of thermodynamics while using both the SGTE description for $\mathrm{T}>298.15 \mathrm{~K}$ and $\mathrm{ab}$ initio calculations at $\mathrm{T}=0 \mathrm{~K}$.

It should be emphasized that, for the sake of zero-point vibrational entropy, Eq 2 is applicable on the temperaturedependence of not only lattice stability, but also the formation energy of stoichiometric compounds and excess energy of solution phases. The abundant well assessed thermodynamic parameters in the existing second generation CALPHAD are valuable. During the development of third generation one may start the assessment of a new chemical system with direct parameter transition into the format of third generation description by using Eq 2. It is worthy to discuss and demonstrate how to apply Eq 2 with a real system i.e. the unary $\mathrm{Al}$ in different cases where the phase is metastable or unstable. In the original paper by Hillert and Selleby ${ }^{[13]}$ the choice of characteristic values $T_{1}$ and $n$ in Eq 2 has not been sufficiently discussed. In this paper, we examine the applicability of the expression of Eq 2. First, we 
test various $T_{1}$ and $n$ and then optimize the values using $a-b T$ at high temperatures and ab initio results at $0 \mathrm{~K}$. As case studies, the lattice stabilities of the hcp and bcc Al were chosen and the information of $a-b T$ was taken from the SGTE database. From these trials, we propose a modified approach to express the lattice stability, Gibbs energy of formation of stoichiometric compounds and excess energy of solution phases from $0 \mathrm{~K}$ to high temperatures that were described by a linear expression in existing database.

In summary, the present paper deals with a methodology on the development of third generation CALPHAD database coupling the information at high temperatures from the second generation and $0 \mathrm{~K}$ from ab initio calculations. The utilized modelling quantity is Gibbs energy. Many efforts have been made to bring in more physical factors into such thermodynamic modelling in the development of third generation CALPHAD database. However, it should be clarified that there is neither any ambitions/attempts nor feasible to develop a pure physical model for Gibbs energy descriptions and consequently phase equilibria predictions. To better demonstrate the methodology meanwhile not lost the generality, the case study on unary Al was presented here for metastable hcp and unstable bcc.

\section{Thermodynamic Models}

\subsection{The Generalized Lattice Stability}

Hillert and Selleby ${ }^{[13]}$ have proposed Eq 2 and also suggested the combination of $n=2$ and 3 , as written by ${ }^{\circ} G^{\beta}-$ ${ }^{\circ} G^{\alpha}=a-0.5 b\left\{T+\frac{T_{1}}{2}\left(1-\frac{T}{T_{1}}\right)^{2}\right\}-0.5 b\left\{T+\frac{T_{1}}{3}\left(1-\frac{T}{T_{1}}\right)^{3}\right\}$, for replacing the $a-b T$ expression. ${ }^{[13]}$ In the present paper, the notation "HS" is used for this original method proposed by Hillert and Selleby. Taking the ratio of the second $(n=2)$ and third $(n=3)$ terms in this equation as a variable $x$, the HS method is extended giving the following generalized expression:

$$
\begin{aligned}
{ }^{\circ} G^{\beta}-{ }^{\circ} G^{\alpha}= & a-x b\left\{T+\frac{T_{1}}{2}\left(1-\frac{T}{T_{1}}\right)^{2}\right\} \\
& -(1-x) b\left\{T+\frac{T_{1}}{3}\left(1-\frac{T}{T_{1}}\right)^{3}\right\} \\
& =A+B T^{2}+C T^{3},
\end{aligned}
$$

where

$$
\begin{aligned}
& A=a-\frac{1}{6}(x+2) b T_{1}, \\
& B=-\frac{1}{2}(2-x) b \frac{1}{T_{1}},
\end{aligned}
$$

$C=\frac{1}{3}(1-x) b \frac{1}{T_{1}^{2}}$.

Equation 3 ensures that the entropy is zero at $0 \mathrm{~K}$. This equation reduces to $a-b T$ at $T=T_{1}$.

\subsection{Low Temperature Description Below Liquidus}

In the third generation of CALPHAD databases, ${ }^{[5,9-11]}$ the temperature dependence of specific heat for pure elements up to the melting point consists of the Einstein function, several power series of temperature and the magnetic contribution in the case where it is applicable:

$C_{P}^{\varphi}=3 R\left(\frac{\theta_{E}}{T}\right)^{2} \frac{e^{\theta_{E} / T}}{\left(e^{\theta_{E} / T}-1\right)^{2}}+c T+\sum_{i} d_{i} T^{i}+C_{P}^{m a g}$.

The first term is the contribution from the harmonic lattice vibration, where $\theta_{E}$ and $R$ represent Einstein temperature and gas constant, respectively. The second term describes the contribution from the electronic excitations and loworder anharmonic corrections. The third term represents contribution from the high-order anharmonic lattice vibrations, where different power series may be used, e.g., $i=2,3$ and/or $4 .^{[2,5,43]}$ The last term is the magnetic contribution, which is zero for pure $\mathrm{Al}$ since this element does not show any magnetic ordering. ${ }^{[1,18]}$ Then, the Gibbs energy expression is derived as follows:

$$
\begin{aligned}
G^{\varphi}= & E_{0}+\frac{3}{2} R \theta_{E}+3 R T \ln \\
& \times\left\{1-\exp \left(-\frac{\theta_{E}}{T}\right)\right\}-\frac{c}{2} T^{2}-\sum_{i} \frac{d_{i}}{i(i+1)} T^{i+1} .
\end{aligned}
$$

Accordingly, the Gibbs energy of the $\beta$ phase relative to the $\alpha$ phase is expressed as follows:

$$
\begin{aligned}
{ }^{\circ}{ }^{\beta}-{ }^{\circ} G^{\alpha}= & \left(E_{0}^{\beta}-E_{0}^{\alpha}\right)+\frac{3}{2} R\left(\theta_{E}^{\beta}-\theta_{E}^{\alpha}\right) \\
& +3 R T\left[\ln \left\{1-\exp \left(-\frac{\theta_{E}^{\beta}}{T}\right)\right\}\right. \\
& \left.-\ln \left\{1-\exp \left(-\frac{\theta_{E}^{\alpha}}{T}\right)\right\}\right]-\frac{1}{2}\left(c^{\beta}-c^{\alpha}\right) T^{2} \\
& -\left(\sum_{i} \frac{d_{i}^{\beta}}{i(i+1)} T^{i+1}-\sum_{j} \frac{d_{j}^{\alpha}}{j(j+1)} T^{j+1}\right) .
\end{aligned}
$$

Applying the expression of $\mathrm{Eq} \mathrm{3,} \mathrm{the} \mathrm{lattice} \mathrm{stability} \mathrm{is}$ represented as: 


$$
\begin{aligned}
& { }^{\circ} G^{\beta}-{ }^{\circ} G^{\alpha}=\frac{3}{2} R\left(\theta_{E}^{\beta}-\theta_{E}^{\alpha}\right) \\
& +3 R T\left[\ln \left\{1-\exp \left(-\frac{\theta_{E}^{\beta}}{T}\right)\right\}\right. \\
& \left.-\ln \left\{1-\exp \left(-\frac{\theta_{E}^{\alpha}}{T}\right)\right\}\right]+A+B T^{2}+C T^{3},
\end{aligned}
$$

where

$$
\begin{aligned}
& A=a^{\prime}-\frac{1}{6}(x+2) b^{\prime} T_{1}, \\
& B=-\frac{1}{2}(2-x) b^{\prime} \frac{1}{T_{1}}, \\
& C=\frac{1}{3}(1-x) b^{\prime} \frac{1}{T_{1}^{2}} .
\end{aligned}
$$

Note that the values of $a^{\prime}$ and $b^{\prime}$ are different from those of $a$ and $b$ because of the Einstein function. The parameters $A$, $B$ and $C$ correspond to the Gibbs energy difference attributed to $E_{0}$, to that of the electronic excitations and loworder anharmonic corrections, and to that of the high-order anharmonic lattice vibrations, respectively. All these parameters are optimized using the PARROT module in the Thermo-Calc software ${ }^{[44]}$ to fit the Gibbs energies above room temperatures calculated by $a-b T$ in the existing second generation databases and the $E_{0}$ values from ab initio calculations available in existing literature.

\subsection{High Temperature Description Above Liquidus}

In the higher temperature range above the melting point where the solid phase is unstable/metastable, the following two expressions (for $C_{P}$ and $G$ ) are used for modelling the solid phase in the third generation of CALPHAD databases. ${ }^{[5,9-11]}$

$$
C_{P}^{\varphi}=3 R\left(\frac{\theta_{E}}{T}\right)^{2} \frac{e^{\theta_{E} / T}}{\left(e^{\theta_{E} / T}-1\right)^{2}}+e+f T^{-6}+g T^{-12}+C_{P}^{m a g}
$$

$$
\begin{aligned}
G^{\varphi}= & \frac{3}{2} R \theta_{E}+3 R T \ln \left\{1-\exp \left(-\frac{\theta_{E}}{T}\right)\right\}+H^{\prime}-S^{\prime} T \\
& +e T(1-\ln T)-\frac{f}{30} T^{-5}-\frac{g}{132} T^{-11} \\
& -\int_{0}^{T}\left\{\int_{0}^{T} \frac{C_{P}^{m a g}}{T} d T\right\} d T .
\end{aligned}
$$

In the present work we use the same power series as in Eq 12 for the polynomial function in the lattice stability at high temperatures above the melting point, giving the following equation:

$$
\begin{aligned}
{ }^{\circ} G^{\beta}-{ }^{\circ} G^{\alpha}= & \frac{3}{2} R\left(\theta_{E}^{\beta}-\theta_{E}^{\alpha}\right)+3 R T\left[\ln \left\{1-\exp \left(-\frac{\theta_{E}^{\beta}}{T}\right)\right\}\right. \\
& \left.-\ln \left\{1-\exp \left(-\frac{\theta_{E}^{\alpha}}{T}\right)\right\}\right]+I+J T \\
& +K T \ln T+L T^{-5}+N T^{-11} \\
& -\left(\int_{0}^{T}\left\{\int_{0}^{T} \frac{C_{P}^{m a g}, \beta}{T} d T\right\}-\int_{0}^{T}\left\{\int_{0}^{T} \frac{C_{P}^{m a g}, \alpha}{T} d T\right\}\right) d T .
\end{aligned}
$$

The parameters $I, J, K, L$ and $N$ are determined to fulfill the conditions that the Gibbs energy, entropy, enthalpy, specific heat and first temperature derivative of specific heat taken from the low-temperature Eq 10 and high-temperature Eq 13 have identical values at the melting temperature. That is the same criteria employed in the development of third generation databases for the unary systems. ${ }^{[5,9-11]}$

\section{Results and Discussion}

We demonstrate how to apply the Eq 10 to approximate metastable lattice stabilities through a case study of $\mathrm{Al}$, in which the fcc structure is stable up to its melting point (934 K). The SGTE unary description was assessed based on thermodynamic and phase stability of corresponding unary and relevant higher order systems, which indeed forms the basis of the second generation databases. The Gibbs energies of hcp and bcc phases relative to the stable fcc phase at $298.15 \mathrm{~K}$ and $10^{5} \mathrm{~Pa}$ are expressed as ${ }^{[1]}$.

$$
{ }^{\circ} G_{A l}^{h c p}-{ }^{\circ} G_{A l}^{f c c}=5481-1.8 \mathrm{~T} \quad(298.15 K<T<2900 K),
$$

${ }^{\circ} G_{A l}^{b c c}-{ }^{\circ} G_{A l}^{f c c}=10083.4-4.813 \mathrm{~T}$

$(298.15 K<T<2900 K)$.

In the following sections, first we show the method for determining the lattice stability of the metastable hcp Al, for which the Debye temperature is known. Secondly, the lattice stability of the (dynamically unstable) bcc phase in $\mathrm{Al}$ is shown as a case for which the Debye temperature is undetermined.

\subsection{Lattice Stability of hep Al}

Contributions from the anharmonic lattice vibration and electronic excitation are rather small and thus the Debye temperature is particularly important at temperatures lower than the Debye temperature $\theta_{D}$. The $\theta_{D}$ can be calculated from experimental or theoretical information of the equilibrium Wigner-Seitz radius $r_{0}$, bulk modulus $B$ and elastic 
constants (and then Poisson's ratio $v$ ), which can be represented as ${ }^{[45-49]}$.

$\theta_{D}(-3)=k(v) \frac{\hbar}{k_{B}}\left(48 \pi^{5}\right)^{1 / 6} \sqrt{\frac{r_{0} B}{M}}$

where

$k(v)=\left[\frac{1}{3}\left\{\left(\frac{1+v}{3(1-v)}\right)^{3 / 2}+2\left(\frac{2(1+v)}{3(1-2 v)}\right)^{3 / 2}\right\}\right]^{-1 / 3}$

and $\hbar, k_{B}$ and $M$ are reduced Planck constant (Dirac constant), Boltzmann constant and atomic weight, respectively. $\theta_{D}(-3)$ means the low temperature limit of the Debye temperature. ${ }^{[47]}$ It has been reported that the hcp structure has $r_{0}=0.1582 \mathrm{~nm}, B=75.02 \mathrm{GPa}$ and the bcc structure has $r_{0}=0.1578 \mathrm{~nm}$ and $B=78.36 \mathrm{GPa}$ from ab initio calculations. ${ }^{[40]}$ Assuming the same values for $v$ and $\theta_{D}(0) / \theta_{D}(-3)$ ratio for the fcc and hep structures, and using $\theta_{E}^{f c c}=294 \mathrm{~K},{ }^{[50]}$ the Einstein temperature that is proportional to $\theta_{D}(0),{ }^{[51]}$ of the hcp $\mathrm{Al}$ is estimated to be $\theta_{E}^{h c p}=288 \mathrm{~K}$.

In the following paragraph, we test how the HS model works for the thermodynamic properties including the effect of the $T_{1}$ temperature. To determine the parameters $a^{\prime}$ and $b^{\prime}$ in Eq $4^{\prime}-6^{\prime}$, the sum of the first and second terms of Eq 10 are approximated by a linear expression in a specific temperature range as follows:

$$
\begin{aligned}
& \frac{3}{2} R\left(\theta_{E}^{\beta}-\theta_{E}^{\alpha}\right) \\
& \quad+3 R T\left[\ln \left\{1-\exp \left(-\frac{\theta_{E}^{\beta}}{T}\right)\right\}-\ln \left\{1-\exp \left(-\frac{\theta_{E}^{\alpha}}{T}\right)\right\}\right] \\
& \quad=a^{\prime \prime}-b^{\prime \prime} T .
\end{aligned}
$$

Then, the lattice stability is re-written as

$$
{ }^{\circ} G^{\beta}-{ }^{\circ} G^{\alpha}=a^{\prime \prime}-b^{\prime \prime} T+A+B T^{2}+C T^{3}(\approx a-b T) .
$$

Therefore, the corresponding linear expression $\left(a^{\prime}-b^{\prime} T\right)$ for $\mathrm{HS} \quad\left(A+B T^{2}+C T^{3}\right)$ is $\left(a-a^{\prime \prime}\right)-$ $\left(b-b^{\prime \prime}\right) T$. In the present case of unary $\mathrm{Al}$, the Einstein temperatures, $\theta_{E}^{f c c}=294 \mathrm{~K}$ and $\theta_{E}^{h c p}=288 \mathrm{~K}$, yield $a^{\prime \prime}-b^{\prime \prime} T=-7.9-0.547 T(298 \mathrm{~K}<T<2900 \mathrm{~K})$ for the linear approximation in Eq 18. Consequently, we obtain $\left(a-a^{\prime \prime}\right)-\left(b-b^{\prime \prime}\right) T=(5481+7.9)-(1.8-0.547)$ $T=5488.9-1.253 T$, which corresponds to the set of $\left(a^{\prime}=5488.9, \quad b^{\prime}=1.253\right)$. Figure 1 shows (a) Gibbs energy, (b) entropy, (c) enthalpy and (d) specific heat of the hcp phase relative to the fcc phase in $\mathrm{Al}$, calculated by using the SGTE expression $a-b T$ or using Eq 10 with different $T_{1}$ temperatures ranging from 834 to $1234 \mathrm{~K}$ and $x=0.5$. Strong deviations are observed at high temperatures for all properties and the HS cannot be readily applied to all temperature ranges. It is obvious that the curvatures at high temperatures are sensitive to the selected $T_{1}$ value. In addition, it is expected that the ratio of $n=2$ and $n=3$ terms (i.e., the value of $x$ in Eq 3-6) affects the description of these thermodynamic properties.

The parameters $A, B$ and $C$ in Eq 10, which are functions of $T_{1}, x, a^{\prime}$, and $b^{\prime}$, were optimized to fit the Gibbs energies calculated by $a-b T$ in the temperature range from $298.15 \mathrm{~K}$ to $T_{\max }\left(T_{\max }: 1934-7034 \mathrm{~K}\right)$. The obtained parameters are listed in Table 1 and the Gibbs energy, entropy, enthalpy and specific heat of the hcp phase relative to the fcc phase are shown in Fig. 2, together with those from $a-b T$. During the optimization, the Einstein temperature of the hcp $\mathrm{Al}$ is fix at $\theta_{E}^{h c p}=288 \mathrm{~K}$.

The HS tells us the relation between the optimized curve and $a-b T$. In the generalized model Eq 10 and $4^{\prime}-6^{\prime}$, the parameters $a^{\prime}, T_{1}$ and $x$ can be calculated according to the optimized value of $A, B$ and $C$ (see Table 1), $b^{\prime}$ value being fixed to be 1.253 . It is interesting to note that $x$ varies in the range of 0.4 and 0.5 when the Gibbs energy calculated by $a-b T$ is considered between $298.15 \mathrm{~K}-T_{\text {max }} \mathrm{K}$, which is close to 0.5 suggested in the original HS model, ${ }^{[13]}$ and that $a^{\prime}$ is close to 5488.9. The $T_{1}$ temperature increases with increasing $T_{\max }$. The trend of shift in each thermodynamic property against the $T_{1}$ temperature is similar between Fig. 1 and 2. As shown in Fig. 2(a), the Gibbs energy decreases with the increase in the $T_{\text {max }}$ temperature and the deviation from $a-b T$ becomes larger at low temperatures. It demonstrates how the choice of different $T_{\text {max }}$ affects the zero point energy.

The difference in $E_{0}$ values between the hcp and fcc structures predicted by ab initio ${ }^{[40,52-55]}$ are also shown in Fig. 2(a) and (c). These data differ by up to about $2000 \mathrm{~J} / \mathrm{mol}$. If we try to fit the highest value of the ab initio calculation by Boettger and Trickey, ${ }^{[52]}$ it turns out that $T_{\text {max }}=2900 \mathrm{~K}$ (the green curve) is the optimal choice. However, we prefer to choose $E_{0}=3333.7 \mathrm{~J} / \mathrm{mol}$ obtained by fitting the total energies reported by Mishra ${ }^{[40]}$ to the Murnaghan equation of the state ${ }^{[56]}$ because it is consistent with the prediction of Debye temperature mentioned above.

For the case of hcp Al, if we try to fit the Gibbs energy data calculated by SGTE's $a-b T$ in the full temperature range between $298.15 \mathrm{~K}$ and $T_{\max } \mathrm{K}$, the resulted $E_{0}$ value seems overestimated compared to the ab initio calculations, even the $T_{\max }$ temperature is set as high as $7034 \mathrm{~K}$. Conversely, the choice of $E_{0}=3333.7 \mathrm{~J} / \mathrm{mol}$ implies the poor fitting of Gibbs energy to SGTE's $a-b T$ at low temperatures. Therefore, during the optimization the low-temperature Gibbs energies were given a low weight and the 

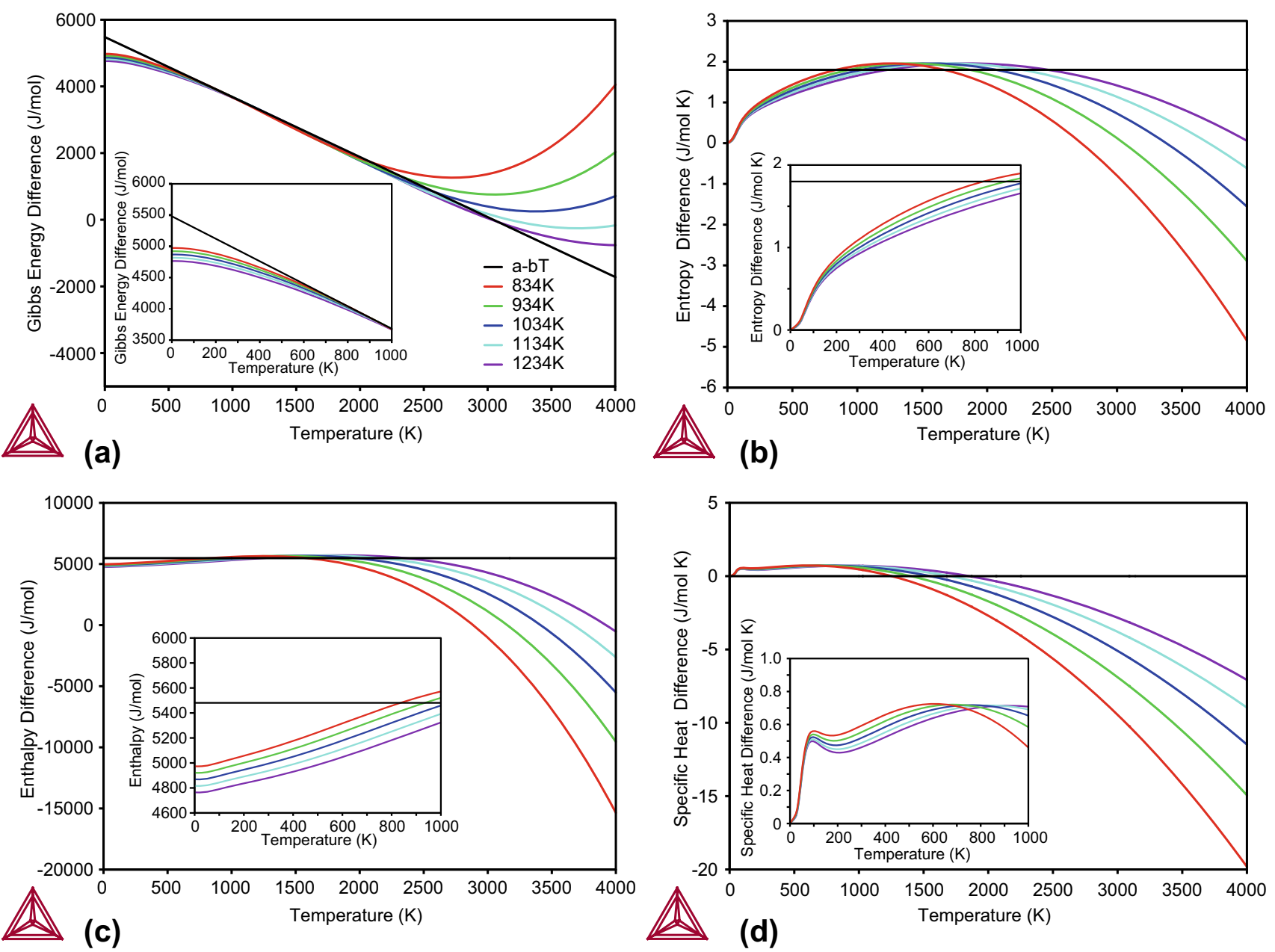

Fig. 1 (a) Gibbs energy difference, (b) entropy difference, (c) enthalpy difference and (d) specific heat difference of hop $\mathrm{Al}$ relative to fcc Al calculated using Eq 10 with various $T_{1}$ temperatures (834-

$1234 \mathrm{~K}$ ) in the temperature range from 0 to $4000 \mathrm{~K}$. Insets depict the figures at low temperatures

Table 1 Optimized parameters $A, B$ and $C$ in Eq 10 for hep Al, where the Gibbs energies calculated by $a-b T$ at $298.15 \mathrm{~K}-T_{\max }\left(T_{\max }: 1934 \mathrm{~K}-\right.$ $7034 \mathrm{~K})$ or at $1527 \mathrm{~K}-T_{\max }$ $\left(T_{\max }: 4000 \mathrm{~K}\right)$ were used in the optimization process

\begin{tabular}{lccccrc}
\hline$T_{\max }, \mathrm{K}$ & $A$ & $B$ & $C$ & $a^{\prime}$ & $T_{1}$ & $x$ \\
\hline 1934 & $5.0890249 \mathrm{E}+03$ & $-1.1328053 \mathrm{E}-03$ & $3.1038079 \mathrm{E}-07$ & 5526.2 & 850.0 & 0.463 \\
2900 & $4.8600006 \mathrm{E}+03$ & $-7.0762739 \mathrm{E}-04$ & $1.2144600 \mathrm{E}-07$ & 5560.8 & 1365.0 & 0.458 \\
3934 & $4.6221173 \mathrm{E}+03$ & $-5.3254152 \mathrm{E}-04$ & $7.0471289 \mathrm{E}-08$ & 5563.8 & 1872.1 & 0.409 \\
4934 & $4.4730527 \mathrm{E}+03$ & $-4.6536643 \mathrm{E}-04$ & $5.3695993 \mathrm{E}-08$ & 5549.5 & 2135.7 & 0.414 \\
7034 & $4.0960759 \mathrm{E}+03$ & $-3.4961941 \mathrm{E}-04$ & $2.9053623 \mathrm{E}-08$ & 5502.3 & 2702.0 & 0.492 \\
4000 & $3.4170455 \mathrm{E}+03$ & $-1.5938254 \mathrm{E}-04$ & $-9.3683979 \mathrm{E}-09$ & 5490.6 & 3089.3 & 1.214 \\
\hline
\end{tabular}

Corresponding values of $a^{\prime}, T_{1}$ and $x$ were determined for $b^{\prime}=1.253$

parameters were optimized mainly using the Gibbs energies at temperatures between $1527 \mathrm{~K}$ (the hcp/bcc transformation temperature in the SGTE database) and $4000 \mathrm{~K}$ as well as $E_{0}=3333.7 \mathrm{~J} / \mathrm{mol}$ from the ab initio calculation. ${ }^{[40]}$ The resultant $E_{0}$ is close to the predicted value by the ab initio calculation. It is also found that the ratio $x$ is very different from 0.5 when the lattice stability of SGTE is largely overestimated or underestimated. The Gibbs energy curve falls into a range between that with $n=2$ and that with $n=3$ in Eq 2 when $x$ is between 0 and 1 (see Fig. 1a of Ref 13), but $x=1.214$ in the present case means that the curve is slightly lower out of the case with $n=2$.

Figure 3 shows the Gibbs energy difference between hcp and fcc $\mathrm{Al}$ at $0-4000 \mathrm{~K}$. While the deviation from $a-b T$ is not large at high temperatures for $T_{\max }=3934$, 4934, 7034 and $4000 \mathrm{~K}$, it unreasonably increases at high 

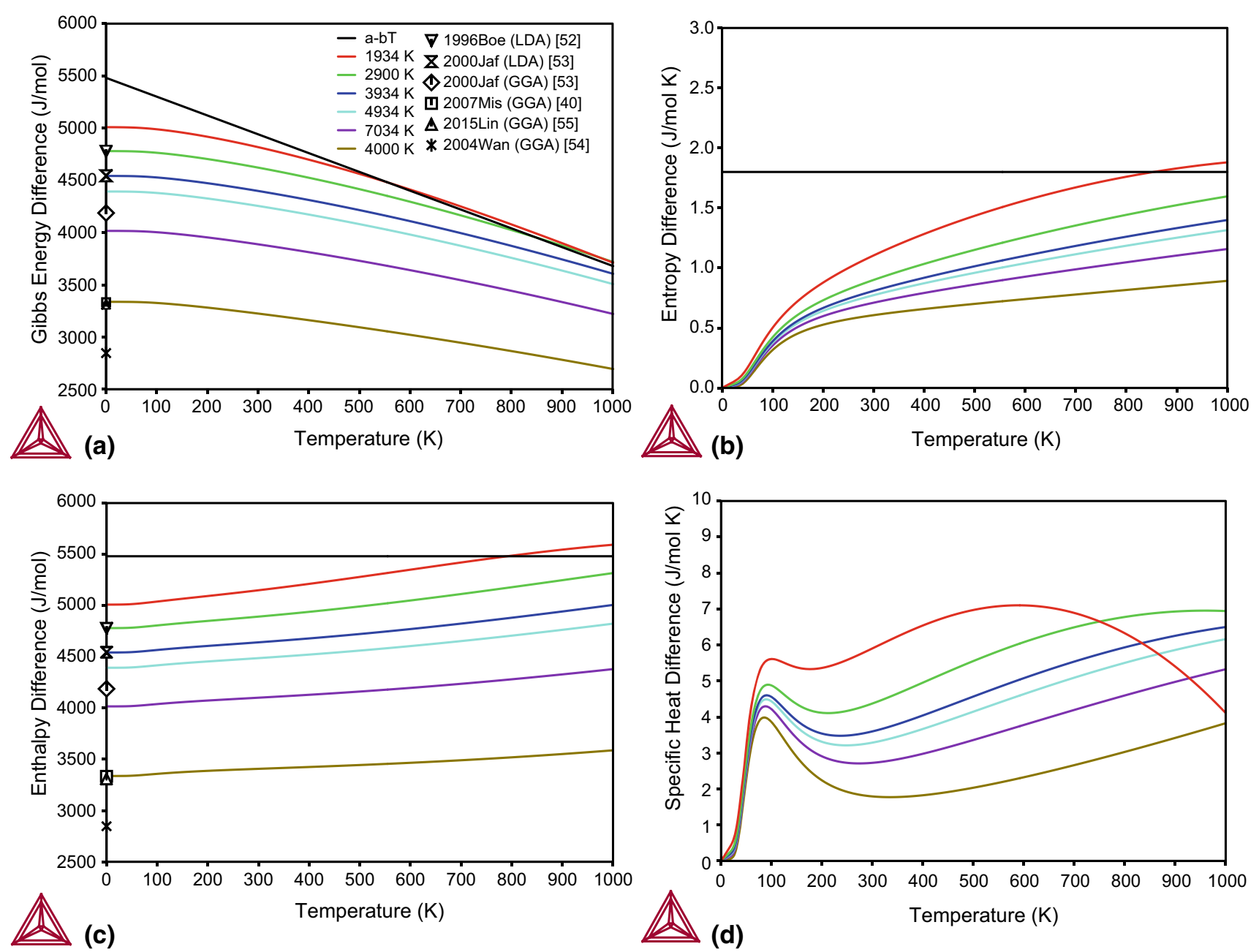

Fig. 2 (a) Gibbs energy difference, (b) entropy difference, (c) enthalpy difference and (d) specific heat difference of hcp Al relative to fcc Al calculated by Eq 10, in which the parameters were optimized

using Gibbs energy data from $a-b T$ at $298 \mathrm{~K}<T<T_{\max }\left(T_{\max }\right.$ : $1934-7034 \mathrm{~K}$ ) or at $1527 \mathrm{~K}<T<4000 \mathrm{~K}$ denoted as $4000 \mathrm{~K}$

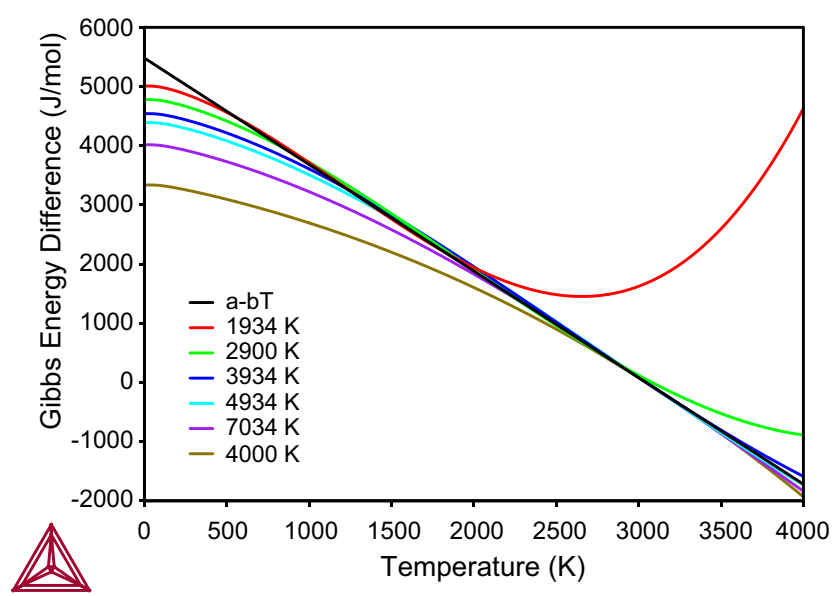

Fig. 3 Gibbs energy difference of hcp Al relative to fcc Al at temperatures from 0 to $4000 \mathrm{~K}$ calculated by $\mathrm{Eq} 10$ beyond the melting temperature, in which the parameters were optimized using Gibbs energy data from $a-b \mathrm{~T}$ at $298<\mathrm{T}<T_{\max } \quad\left(T_{\max }\right.$ : $1934-7034 \mathrm{~K}$ ) or at $1527<\mathrm{T}<4000 \mathrm{~K}$ denoted as $4000 \mathrm{~K}$

temperatures for $T_{\max }=1934$ and $2900 \mathrm{~K}$. In such cases, another expression, Eq 13, for the high temperature range is necessary. The partitioning of the Gibbs energy description into two temperature ranges is discussed in the following section for the case of bcc Al.

Considering the $E_{0}$ value, the expression for $T_{\max }=4000 \mathrm{~K}$ is recommended in this work. The procedure of determination of lattice stability for hep $\mathrm{Al}$ is summarized as follows: (1) determination of Einstein temperature, (2) optimization of parameters $A-C$ in Eq 10 using the Gibbs energy calculated by SGTE data in a selected temperature range, (3) determination of appropriate temperature range judged by $E_{0}$, and (4) calculation of parameters $I, J, K, L$ and $N$ in Eq 13 for high temperature range, if necessary. 

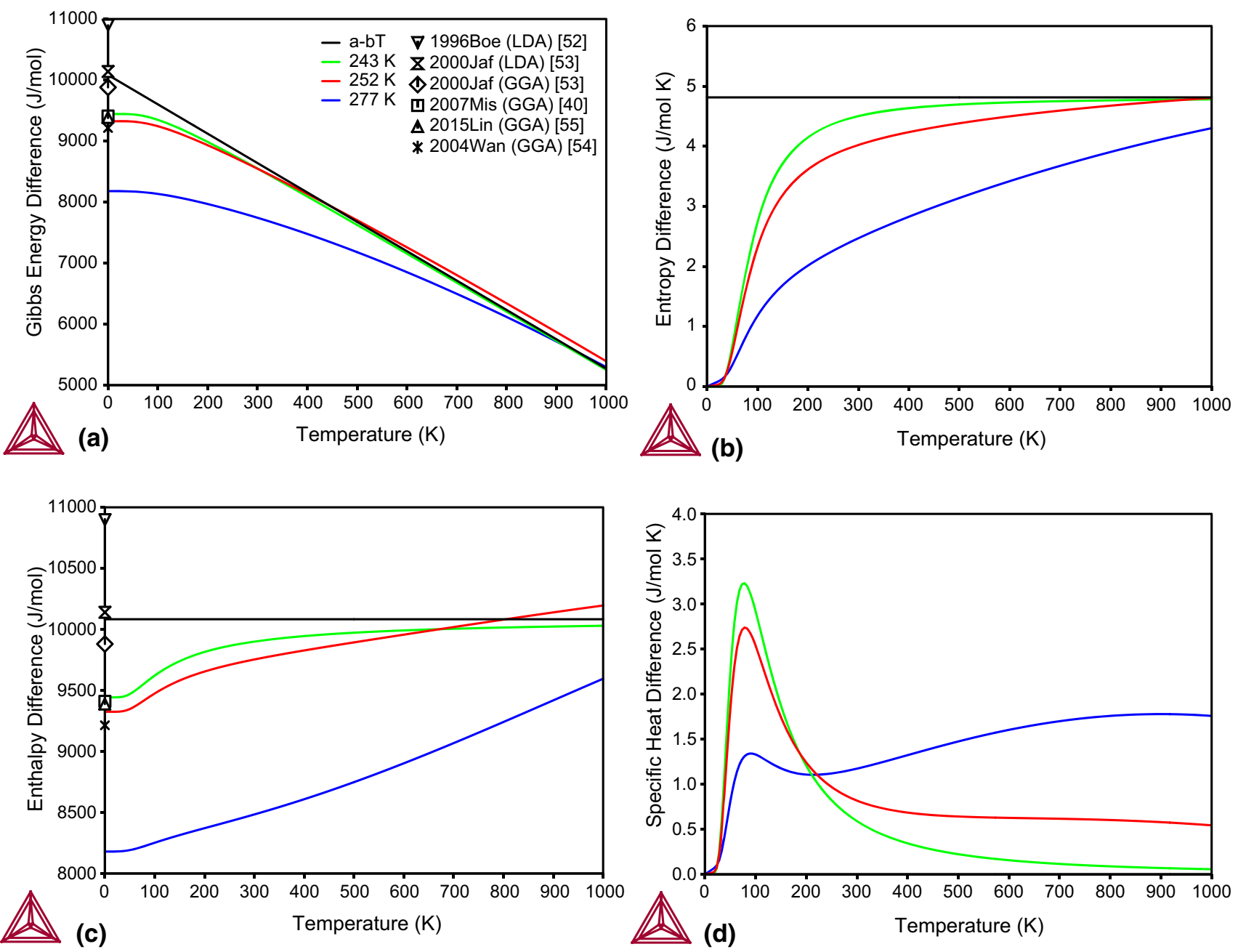

Fig. 4 (a) Gibbs energy difference, (b) entropy difference, (c) enthalpy difference and (d) specific heat difference of bcc $\mathrm{Al}$ relative to fcc Al calculated by Eq 10 with different Einstein temperatures

\subsection{Lattice Stability of bcc Al}

This is a case in which the Debye temperature is unknown or unreliable for some reason, such as limited information. The bcc $\mathrm{Al}$ is dynamically unstable at $0 \mathrm{~K}$ under ambient pressure. ${ }^{[25-28]}$ Although the Debye temperature is not definable and the entropy has no physical meaning for an unstable phase, one needs to determine the lattice stability in the CALPHAD method. One option is that we define the lattice stability by changing $a-b T$ of the SGTE form using Eq 3 without the Einstein model. Here, we tried expressing the lattice stability of bcc $\mathrm{Al}$ using the Einstein model, which is consistent with other structures. An empirical rule for predicting the Debye temperature for a dynamically unstable structure has been proposed, ${ }^{[49]}$ but reasonable result of Gibbs energy at finite temperatures was not obtained for the bcc Al.

If the Poisson ratio of the bcc structure is assumed to be the same as the fcc structure, the Einstein temperature $\theta_{E}$ is calculated equal to $277 \mathrm{~K}$. The parameters were optimized using the SGTE Gibbs energy $a-b T$ at $298-2900 \mathrm{~K}$ and the result shows that the $E_{0}$ is lower than the ab initio calculation results (Fig. 4), implying that the estimated Einstein temperature is too low.

Therefore, the Einstein temperature $\theta_{E}$ for the bcc $\mathrm{Al}$ was optimized as well as other parameters in Eq 10. In the first trial, only the Einstein function was optimized using the SGTE Gibbs energy $a-b T$ at 298-2900 K and $E_{0}$, ${ }^{[40]}$ and the $\theta_{E}$ became about $243 \mathrm{~K}$. Although the fit is good, as shown in Fig. 4, the difference in anharmonic and electronic excitation is not considered; thus, this $\theta_{E}$ may be considered as the lowest limit. Thereafter both the Einstein temperature $\theta_{E}$ and the parameters $A, B$ and $C$ were optimized to fit the ab initio calculated $E_{0},{ }^{[40]}$ the SGTE Gibbs energy by $a-b T$ at $298-2900 \mathrm{~K}$, and the data point of specific heat at $300-900 \mathrm{~K}$ from the calculation for $\theta_{E}^{b c c}=277 \mathrm{~K}$. The optimized $\theta_{E}^{b c c}$ is $252 \mathrm{~K}$ and the $E_{0}$ agrees with the ab initio result. ${ }^{[40]}$ Moreover, the difference 
Table 2 Optimized parameters $A, B, C$ and $\theta_{E}$ in Eq 10 for bcc $\mathrm{Al}$

\begin{tabular}{lllllll}
\hline$A$ & \multicolumn{1}{c}{$B$} & \multicolumn{1}{c}{$C$} & \multicolumn{1}{c}{$\theta_{E}$} & \multicolumn{1}{c}{$a^{\prime}$} & \multicolumn{1}{c}{$T_{1}$} & \multicolumn{1}{c}{$x$} \\
\hline $1.0086884 \mathrm{E}+04$ & $\ldots$ & $\ldots$ & $2.4279542 \mathrm{E}+02$ & $\ldots$ & $\ldots$ & $\ldots$ \\
$9.8592063 \mathrm{E}+03$ & $-6.5847880 \mathrm{E}-04$ & $1.3685578 \mathrm{E}-07$ & $2.5157826 \mathrm{E}+02$ & 10246.6 & 1005.3 & 0.544 \\
$8.3967406 \mathrm{E}+03$ & $-1.9209886 \mathrm{E}-03$ & $3.5071832 \mathrm{E}-07$ & 277 & 10214.1 & 1381.9 & 0.391 \\
\hline
\end{tabular}

The difference in the Einstein function is approximated in the temperature range from 298 to $2900 \mathrm{~K}$ to be $a^{\prime \prime}-b^{\prime \prime} T=-49.7-3.904 T\left(\theta_{E}^{f c c}=294 \mathrm{~K}, \quad \theta_{E}^{b c c}=252 \mathrm{~K}\right) \quad$ and $\quad a^{\prime \prime}-b^{\prime \prime} T=-21.2-1.513 T \quad\left(\theta_{E}^{f c c}=\right.$ $\left.294 \mathrm{~K}, \theta_{E}^{b c c}=277 \mathrm{~K}\right)$ and the linear approximation for HS is written as $\left(a-a^{\prime \prime}\right)-\left(b-b^{\prime \prime}\right)=10133.1-$ $0.909 \mathrm{~T}\left(\theta_{E}^{b c c}=252 \mathrm{~K}\right)$ and $\left(a-a^{\prime \prime}\right)-\left(b-b^{\prime \prime}\right) T=10104.6-3.300 T\left(\theta_{E}^{b c c}=277 \mathrm{~K}\right)$. Parameters $a^{\prime}, T_{1}$ and $x$ in HS were calculated for $b^{\prime}=0.909\left(\theta_{E}^{b c c}=252 \mathrm{~K}\right)$ and $b^{\prime}=3.300\left(\theta_{E}^{b c c}=277 \mathrm{~K}\right)$ in Eq $4^{\prime}-6^{\prime}$

Table 3 Thermodynamic description of hcp and bcc Al, relative to fcc Al

\begin{tabular}{ll}
\hline HCP & \\
& $+3.4170455 \mathrm{E}+03-1.5938254 \mathrm{E}-04 * T^{2}-9.3683979 \mathrm{E}-09 * T^{3}+\mathrm{GEIN}(2.88 \mathrm{E}+02) \quad(0 \leq \mathrm{T} \leq 4000)$ \\
$\mathrm{BCC}$ & $+9.8592063 \mathrm{E}+03-6.5847880 \mathrm{E}-04 * T^{2}+1.3685578 \mathrm{E}-07 * T^{3}+\mathrm{GEIN}(2.5157826 \mathrm{E}+02) \quad(0 \leq \mathrm{T} \leq 934)$ \\
& $+1.0373589 \mathrm{E}+04-1.1172748 * T+1.4807155 \mathrm{E}-02 * \mathrm{~T} * \mathrm{LN}(\mathrm{T})-2.2644071 \mathrm{E}+16 * \mathrm{~T}^{-5} \quad(934 \leq \mathrm{T} \leq 4000)$ \\
& $+1.6518853 \mathrm{E}+33 * T^{-11}+\mathrm{GEIN}(2.5157826 \mathrm{E}+02) \quad(2)$ \\
Function & \\
& $\operatorname{GEIN}\left(\theta_{E}\right)=\frac{3}{2} R \theta_{E}+3 R T \ln \left\{1-\exp \left(-\frac{\theta_{E}}{T}\right)\right\}$
\end{tabular}

in the specific heat between $\theta_{E}^{b c c}=243$ and $252 \mathrm{~K}$ gives space to the difference in the electronic and anharmonic contributions, although it is not large. The optimized parameters are summarized in Table 2.

The expression of Eq 10 results in an unreasonable increase in the Gibbs energy at high temperatures, such as $4000 \mathrm{~K}$. In such cases, another expression, such as Eq 13, should be used for the temperature range higher than the melting point of the stable phase. The parameters $I, J, K, L$ and $N$ in Eq 13 for $\theta_{E}^{b c c}=252 \mathrm{~K}$ are listed in Table 3, where these parameters are mathematically determined by the conditions that the Gibbs energy, entropy, enthalpy, specific heat and its first derivative calculated from the low temperature expression $\mathrm{Eq} 10$ and the high temperature expression Eq 13 are equal at the melting point of the stable phase (934 K). The Gibbs energy, entropy, enthalpy and specific heat for $\theta_{D}^{b c c}=252 \mathrm{~K}$ using Eq 10 at lower temperatures and $\mathrm{Eq} 13$ at high temperatures are shown in Fig. 5 in comparison with $a-b T$. Each curve is continuous at the melting point of the fcc $\mathrm{Al}(934 \mathrm{~K})$.

The procedure of determination of lattice stability for bec Al is summarized as follows; (1) optimization of parameters $A-C$ and Einstein temperature in Eq 10 using the Gibbs energy calculated by SGTE data in a selected temperature range, $E_{0}$ and $\mathrm{C}_{\mathrm{P}}$, and (2) calculation of parameters $I, J, K, L$ and $N$ in $\mathrm{Eq} 13$ for a high temperature range, if necessary.

\subsection{Lattice Stabilities of All Allotropes of Unary Aluminum}

The optimized thermodynamic parameters for the hcp and bcc $\mathrm{Al}$ in the present work are summarized in Table 3. Figure 6 shows the Gibbs energy, entropy, enthalpy and specific heat of the hcp and bcc phases relative to the fcc phase in pure Al. The Gibbs energy of the liquid phase ${ }^{[50]}$ is also shown for reference. The lattice stabilities of the hep and bcc $\mathrm{Al}$ recommended in this work are well fitted to those in $\mathrm{SGTE}^{[1]}$ at high temperatures, as shown in Fig. 6(a), and deviation at low temperatures was determined considering the ab initio calculations. ${ }^{[40]}$ The entropy demonstrated in Fig. 6(b) proves to be zero at $0 \mathrm{~K}$. The specific heat of the fcc, ${ }^{[50]}$ hcp and bcc phases is shown in Fig. 7. As mentioned in the introduction, the difference in the specific heat between the fcc and hcp or bcc phases is ignored in the SGTE because of the simple $a-b T$ expression of the lattice stability. Conversely, the specific heat of the bcc Al with lower Einstein temperature has higher value and that of the hcp Al is slightly higher compared with that of the fcc $\mathrm{Al}$ in the present work. In more physical sense, the contributions from the electronic excitation and the anharmonic lattice vibration (corresponding to $\mathrm{T}^{2}$ and $\mathrm{T}^{3}$ terms) should be theoretically considered. A similar way to the SGTE is adopted that the specific heat at high temperatures is close to each other. 

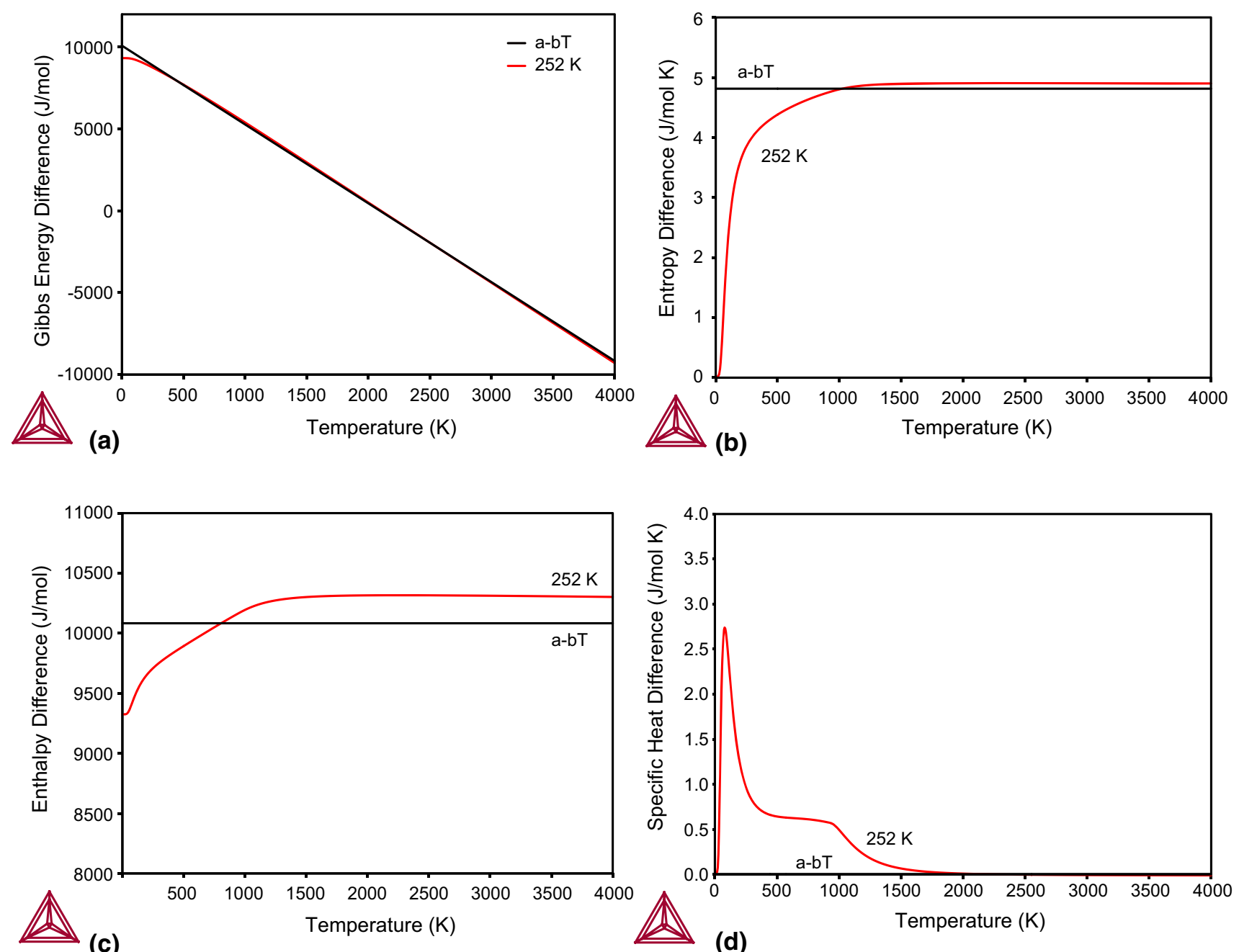

Fig. 5 (a) Gibbs energy difference, (b) entropy difference, (c) enthalpy difference and (d) specific heat difference of bcc Al relative to fcc $\mathrm{Al}$ at temperatures from 0 to $4000 \mathrm{~K}$, where $\mathrm{Eq} 10$ with the

Einstein temperature of $252 \mathrm{~K}$ for bcc $\mathrm{Al}$ is used at lower temperatures (0-934 K) and Eq 13 is used at high temperatures $(934-4000 \mathrm{~K})$

The melting point, enthalpy of transformation and entropy of transformation of the present work are compared with those of the SGTE in Table 4. These thermodynamic properties of the present work are close to those of the SGTE database at high temperatures, i.e., for the fcc/ bcc and fcc/hcp transformations, but noticeably revised at low temperatures, particularly for the transformations involving the hep phase.

\section{Conclusions}

A simple method to assess the lattice stability in the framework of the modelling for the third generation CALPHAD databases was proposed. The expression obeys the third law of thermodynamics, meanwhile, taking full advantage of ab initio calculations at $0 \mathrm{~K}$ and those existing descriptions in the second generation data for

$\mathrm{T}>298 \mathrm{~K}$. The method was demonstrated using the unary $\mathrm{Al}$ system as an example, where the lattice stabilities of the metastable hcp and unstable bcc Al were investigated.

In the case where the Einstein temperature information is available or it can be predicted (e.g., hcp Al), only the other parameters, i.e. A-C in Eq 10, in the HS model for extending the linear function are optimized using the Gibbs energies from the existing SGTE description and the results of ab initio calculations. An appropriate temperature range of the Gibbs energy from SGTE should be chosen for optimization so as to fit other available information, such as $E_{0}$ predicted by ab initio calculation. In the other case that the Einstein temperature is unknown (e.g., bcc $\mathrm{Al}$ ), both the Einstein temperature and HS model parameters are optimized using the Gibbs energies from SGTE and $E_{0}$ predicted by ab initio calculation.

The ratio $x$ in the HS expression is close to 0.5 when the lattice stability of SGTE is acceptable in a certain range of 

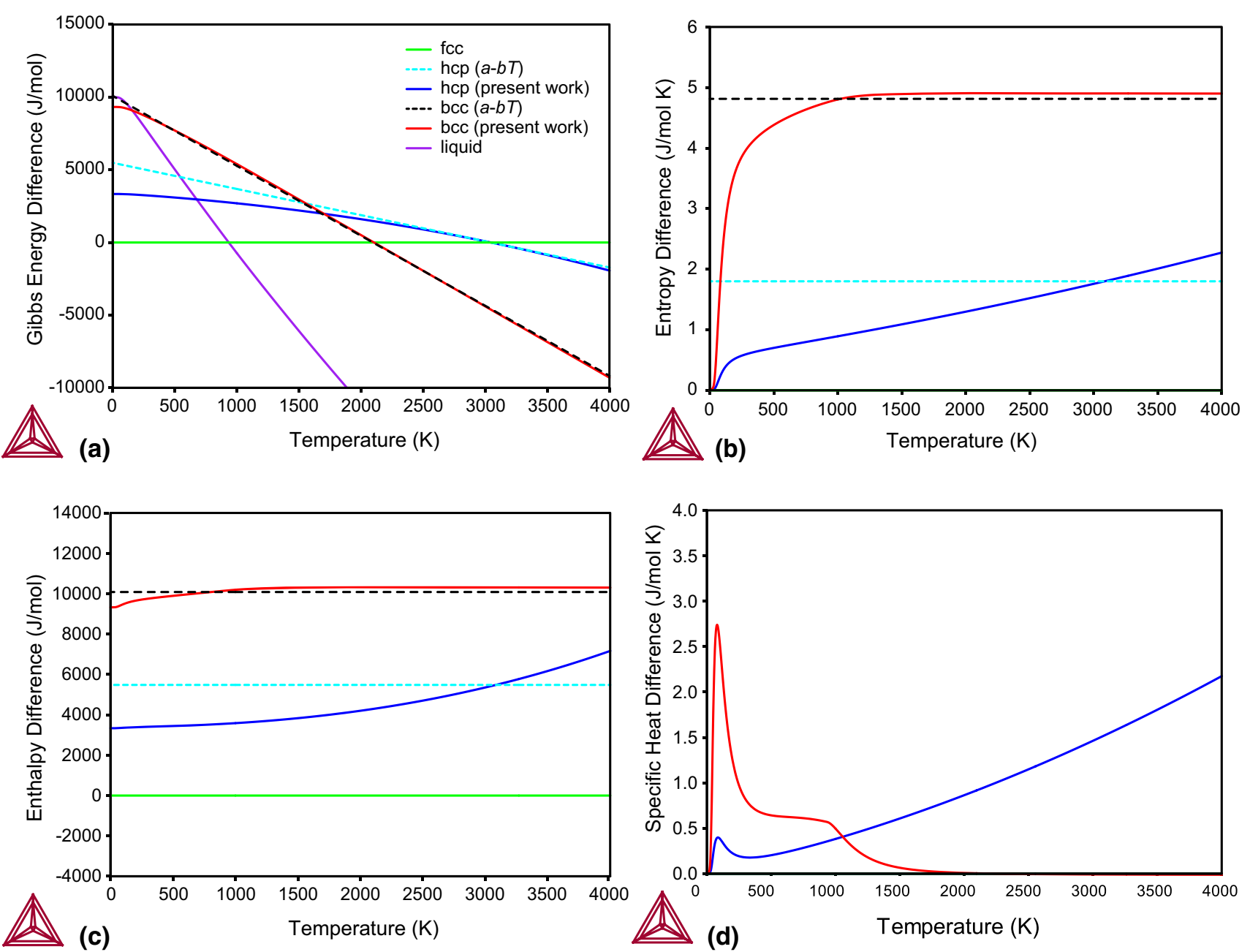

Fig. 6 (a) Gibbs energy difference, (b) entropy difference, (c) enthalpy difference and (d) specific heat difference of hcp, bcc and liquid Al relative to fcc Al calculated by SGTE expression $a-b T$ or descriptions listed in Table 3
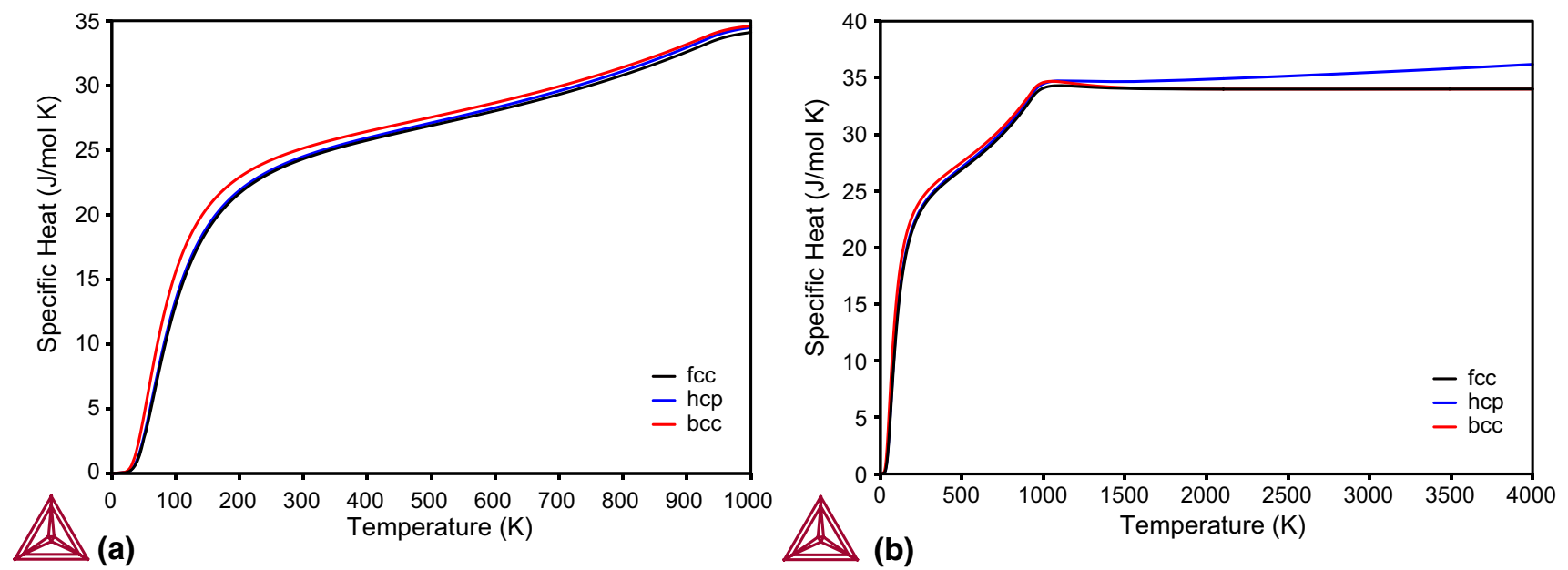

Fig. 7 Specific heat of fcc, hcp and bcc Al calculated by using descriptions listed in Table 3 at temperatures (a) from 0 to $1000 \mathrm{~K}$ and (b) from 0 to $4000 \mathrm{~K}$ 
Table 4 Comparisons of melting temperature, enthalpy of transformation and entropy of transformation between the present work and SGTE in parentheses

\begin{tabular}{lccc}
\hline & $\mathrm{T}_{\mathrm{m}}, \mathrm{K}$ & $\Delta \mathrm{H}_{\text {trans }}, \mathrm{kJ} / \mathrm{mol}$ & $\Delta \mathrm{S}_{\text {trans }}, \mathrm{J} / \mathrm{mol} \mathrm{K}$ \\
\hline $\mathrm{bcc} / \mathrm{liq}$ & $156.7(131.2)$ & $1.200(0.922)$ & $7.660(7.029)$ \\
$\mathrm{hcp} / \mathrm{liq}$ & $675.6(550.2)$ & $7.318(5.517)$ & $10.831(10.026)$ \\
$\mathrm{hcp} / \mathrm{bcc}$ & $1701.1(1527.4)$ & $6.344(4.602)$ & $3.730(3.013)$ \\
$\mathrm{fcc} / \mathrm{bcc}$ & $2103.5(2095.0)$ & $10.316(10.083)$ & $4.904(4.813)$ \\
$\mathrm{fcc} / \mathrm{hcp}$ & $3045.3(3045.0)$ & $5.422(5.481)$ & $1.780(1.800)$ \\
\hline
\end{tabular}

temperature. It is necessary to use a different expression for the Gibbs energy at temperatures higher than the melting point of the stable phase to avoid strong deviation, in which the parameters $I, J, K, L$ and $N$ in Eq 13 can mathematically be determined by the low temperature Eq 10. This generalized method is simple but flexible enough to estimate the lattice stabilities using the existing second generation data down to room temperature, as suggested by SGTE, and ab initio calculations, e.g., total energy at $0 \mathrm{~K}$.

The formation energy of stoichiometric compounds and excess energy of solution phases are often expressed by $a-b T$ in the second generation data especially when thermodynamic information is limited. The present method could be readily applied for these energies to avoid any non-zero vibrational entropy at $0 \mathrm{~K}$.

Acknowledgments The authors are grateful to Prof. Malin Selleby and Prof. Mats Hillert for valuable discussions. The work was performed within the VINN Excellence Center Hero-m, financed by VINNOVA (Grant No. 2012-02892), the Swedish Governmental Agency for Innovation Systems, Swedish industry, and KTH Royal Institute of Technology. One of the authors, TO, acknowledges the support from JSPS KAKENHI and from the Program for Promoting the Enhancement of Research Universities by MEXT, Japan.

Open Access This article is distributed under the terms of the Creative Commons Attribution 4.0 International License (http://crea tivecommons.org/licenses/by/4.0/), which permits unrestricted use, distribution, and reproduction in any medium, provided you give appropriate credit to the original author(s) and the source, provide a link to the Creative Commons license, and indicate if changes were made.

\section{References}

1. A.T. Dinsdale, SGTE Data for Pure Elements, CALPHAD, 1991, 15(4), p 317-425

2. M.W. Chase, I. Ansara, A. Dinsdale, G. Eriksson, G. Grimvall, L. Hoglund, and H. Yokokawa, Workshop on Thermodynamic Models and Data for Pure Elements and Other Endmembers of Solutions: Schlo $\beta$ Ringberg, Feb. 26 to March 3, 1995, CALPHAD, 1995, 19(4), p 437-447

3. J. Ågren, B. Cheynet, M.T. Clavaguera-Mora, K. Hack, J. Hertz, F. Sommer, and U. Kattner, Workshop on Thermodynamic Models and Data for Pure Elements and Other Endmembers of Solutions: Schlo $\beta$ Ringberg, Febr. 21, to March 3, 1995, CALPHAD, 1995, 19(4), p 449-480
4. D. de Fontaine, S.G. Fries, G. Inden, P. Miodownik, R. SchmidFetzer, and S.-L. Chen, Workshop on Thermodynamic Models and Data for Pure Elements and other Endmembers of Solutions: Schlo $\beta$ Ringberg, Febr. 26, to March 3, 1995, CALPHAD, 1995, 19(4), p 499-536

5. Q. Chen and B. Sundman, Modeling of Thermodynamic Properties for Bcc, Fcc, Liquid, and Amorphous Iron, JPE, 2001, 22(6), p 631-644

6. J. Vřšst'ál, J. Štrof, and J. Pavlů, Extension of SGTE Data for Pure Elements to Zero Kelvin temperature-A Case Study, CALPHAD, 2012, 37, p 37-48

7. M. Palumbo, B. Burton, A.C.E. Silva, B. Fultz, B. Grabowski, G. Grimvall, B. Hallstedt, O. Hellman, B. Lindahl, A. Schneider, P.E.A. Turchi, and W. Xiong, Thermodynamic Modelling of Crystalline Unary Phases, Phys. Status Solidi B Basic Solid State Phys., 2014, 251(1), p 14-32

8. I. Roslyakova, B. Sundman, H. Dette, L.J. Zhang, and I. Steinbach, Modeling of Gibbs Energies of Pure Elements Down to $0 \mathrm{~K}$ Using Segmented Regression, CALPHAD, 2016, 55, p 165-180 (in English)

9. S. Bigdeli, H. Ehtehsami, Q. Chen, H. Mao, P. Korzhavy, and M. Selleby, New Description of Metastable HCP Phase for Unaries Fe and Mn: Coupling Between First-Principles Calculations and CALPHAD Modeling, Phys. Status Solidi (b), 2016, 253(9), p $1830-1836$

10. S. Bigdeli, H. Mao, and M. Selleby, On the Third-Generation Calphad Databases: An Updated Description of Mn, Phys. Status Solidi (b), 2015, 252(10), p 2199-2208

11. Z. Li, S. Bigdeli, H. Mao, Q. Chen, and M. Selleby, Thermodynamic Evaluation of Pure Co for the Third Generation of Thermodynamic Databases, Phys. Status Solidi (b), 2017, 254(2), p 1600231-1600231

12. N. Saunders, A.P. Miodownik, and A.T. Dinsdale, Metastable Lattice Stabilities for the Elements, CALPHAD, 1988, 12(4), p 351-374

13. M. Hillert and M. Selleby, Methods for Storage of Gibbs Energy Data of Substances, CALPHAD, 2016, 53, p 146-150

14. T.E. Pochapsky, heat Capacity and Resistance Measurements for Aluminum and Lead Wires, Acta Metall., 1953, 1(6), p 747-751

15. N.E. Phillips, Heat capacity of Aluminum between $0.1^{\circ} \mathrm{K}$ and $4.0^{\circ} \mathrm{K}, 1959, \mathbf{1 1 4}(3), \mathrm{p} 676-685$

16. C.R. Brooks and R.E. Bingham, Specific Heat of Aluminum form 330 to 890 Degrees K and Contributions from Formation of Vacancies and Anharmonic Effects, J. Phys. Chem. Solids, 1968, 29(9), p 1553

17. R.C. Shukla, C.A. Plint, and D.A. Ditmars, Aluminum. II. Derivation of Cv0from $\mathrm{Cp}$ and Comparison to $\mathrm{Cv} 0$ Calculated from Anharmonic Models, Int. J. Thermophys., 1985, 6(5), p 517532

18. P.D. Desai, Thermodynamic Properties of Aluminum, Int. J. Thermophys., 1987, 8(5), p 621-638

19. G.K. Straub, J.B. Aidun, J.M. Wills, C.R. Sanchez-Castro, and D.C. Wallace, Ab Initio Calculation of Melting and Thermodynamic Properties of Crystal and Liquid Aluminum, Phys. Rev. B Condens. Matter., 1994, 50(8), p 5055-5061

20. Y. Wang and L. Li, Mean-Field Potential Approach to Thermodynamic Properties of Metal: $\mathrm{Al}$ as a Prototype, Phys. Rev. B, 2000, 62(1), p 196-202

21. C. Bercegeay and S. Bernard, First-Principles Equations of State and Elastic Properties of Seven Metals, Phys. Rev. B, 2005, 72(21), p 214101

22. A. Debernardi, M. Alouani, and H. Dreysse, Ab Initio Thermodynamics of Metals: Al and W, Phys. Rev. B, 2001, 63(6), p 064305 (in English)

23. M. Forsblom, N. Sandberg, and G. Grimvall, Anharmonic Effects in the Heat Capacity of Al, Phys. Rev. B, 2004, 69(16), p 165106 
24. B. Grabowski, L. Ismer, T. Hickel, and J. Neugebauer, Ab Initio Up to the Melting Point: Anharmonicity and Vacancies in Aluminum, Phys. Rev. B, 2009, 79(13), p 134106

25. G. Grimvall, B. Magyari-Köpe, V. Ozolinš̌, and K.A. Persson, Lattice Instabilities in Metallic Elements, Rev. Mod. Phys., 2012, 84(2), p 945-986

26. L. Weixue and W. Tzuchiang, Ab Initio Investigation of the Elasticity and Stability of Aluminium, J. Phys. Condens. Matter., 1998, 10(43), p 9889

27. L.G. Wang, M. Šob, and Z. Zhang, Instability of Higher-Energy Phases in Simple and Transition Metals, J. Phys. Chem. Solids, 2003, 64(5), p 863-872

28. Y.B. Kudasov, O.M. Surdin, A.S. Korshunov, V.N. Pavlov, N.V. Frolova, and R.S. Kuzin, Lattice Dynamics and Phase Diagram of Aluminum at High Temperatures, J. Exp. Theor. Phys., 2013, 117(4), p 664-671

29. P.J. Craievich, J.M. Sanchez, R.E. Watson, and M. Weinert, Structural Instabilities of Excited Phases, Phys. Rev. B, 1997, $\mathbf{5 5}(2), \mathrm{p}$ 787-797

30. Y. Mishin, D. Farkas, M.J. Mehl, and D.A. Papaconstantopoulos, Interatomic Potentials for Monoatomic Metals from Experimental Data and Ab Initio Calculations, Phys. Rev. B, 1999, 59(5), p 3393-3407

31. G.V. Sin'ko and N.A. Smirnov, Aluminum Under Pressure: Ab Initio Calculations, Phys. Met. Metallogr., 1999, 87(5), p 374378

32. V. Mishra and S. Chaturvedi, Fcc-hep Phase Coexistence in Al at High Pressures: Explanation in Terms of Density of States, Physica B, 2008, 403(17), p 2676-2677

33. C. Friedli and N.W. Ashcroft, Aluminum Under High Pressure. I. Equation of State, Phys. Rev. B, 1975, 12(12), p 5552-5559

34. P.K. Lam and M.L. Cohen, Calculation of High-Pressure Phases of A1, Phys. Rev. B, 1983, 27(10), p 5986-5991

35. A.K. McMahan and J.A. Moriarty, Structural Phase Stability in Third-Period Simple Metals, Phys. Rev. B, 1983, 27(6), p 32353251

36. J.C. Boettger and S.B. Trickey, Total Energy and Pressure in the Gaussian-Orbitals Technique. II. Pressure-Induced Crystallographic Phase Transition and Equilibrium Properties of Aluminum, Phys. Rev. B, 1984, 29(12), p 6434-6442

37. J.C. Boettger and S.B. Trickey, High-Precision Calculation of Crystallographic Phase-Transition Pressures for Aluminum, Phys. Rev. B, 1995, 51(21), p 15623-15625

38. G.V. Sin'ko and N.A. Smirnov, Ab Initio Calculations of Elastic Constants and Thermodynamic Properties of bcc, fcc, and hcp Al Crystals Under Pressure, J Phys. Condens. Matter., 2002, 14(29), p 6989-7005

39. F. Jona and P.M. Marcus, Lattice Parameters of Aluminium in the Mbar Range by First-Principles, J. Phys. Condens. Matter, 2006, 18(48), p 10881
40. V. Mishra and S. Chaturvedi, Theoretical Study of FCC-HCP Phase Coexistence and Phase Stability in Al by FP-LAPW Method with GGA for Exchange and Correlation, Physica B, 2007, 393(1-2), p 278-284

41. M.J. Tambe, N. Bonini, and N. Marzari, Bulk Aluminum at High Pressure: A First-Principles Study, Phys. Rev. B, 2008, 77(17), p 172102

42. S.L. Qiu and P.M. Marcus, Saddle-Point Equilibrium Lines Between fcc and bcc Phases in $\mathrm{Al}$ and $\mathrm{Ca}$ from First Principles, Eur. Phys. J, B, 2013, 86(10), p 425

43. G.T. Furukawa, W.G. Saba, and M.L. Reilly, NSRDS-NBS, 1968, 18, p 1

44. J.O. Andersson, T. Helander, L.H. Hoglund, P.F. Shi, and B. Sundman, THERMO-CALC \& DICTRA, Computational Tools for Materials Science, CALPHAD, 2002, 26(2), p 273-312

45. P. Debye, The Theory of Specific Warmth, Ann. Phys. Berlin, 1912, 39(14), p 789-839 (in German)

46. O.L. Anderson, Physical Acoustics, Acadmic Press, Cambridge, 1965

47. G. Grimvall, Thermophysical Properties of Materialsed, Elsevier, New York City, 1999

48. V.L. Moruzzi, J.F. Janak, and K. Schwarz, Calculated Thermal Properties of Metals, Phys. Rev. B, 1988, 37(2), p 790-799

49. Q. Chen and B. Sundman, Calculation of Debye Temperature for Crystalline Structures-A Case Study on $\mathrm{Ti}, \mathrm{Zr}$, and Hf, Acta Mater., 2001, 49(6), p 947-961

50. S. Bigdeli, L. Zhu, A. Glensk, B. Grabowski, B. Lindahl, M. Selleby (unpublished work)

51. G. Cacciamani, A. Chang, G. Grimvall, P. Franke, L. Kaufman, P. Miodownik, J.M. Sanchez, M. Schalin, and C. Sigli, Workshop on Thermodynamic Modelling of Solutions and Alloys, $C A L$ PHAD, 1997, 21(2), p 219-246

52. J.C. Boettger and S.B. Trickey, High-Precision Calculation of the Equation of State and Crystallographic Phase Stability for Aluminum, Phys. Rev. B, 1996, 53(6), p 3007-3012

53. J.E. Jaffe, R.J. Kurtz, and M. Gutowski, Comparison of Embedded-Atom Models and First-Principles Calculations for Al Phase Equilibrium, Comput. Mater. Sci., 2000, 18(2), p 199-204

54. Y. Wang, S. Curtarolo, C. Jiang, R. Arroyave, T. Wang, G. Ceder, L.Q. Chen, and Z.K. Liu, Ab Initio Lattice Stability in Comparison with CALPHAD Lattice Stability, CALPHAD, 2004, 28(1), p 79-90

55. B. Lindahl, X.L. Liu, Z.-K. Liu, and M. Selleby, A Thermodynamic Re-assessment of $\mathrm{Al}-\mathrm{V}$ Toward an Assessment of the Ternary Al-Ti-V System, CALPHAD, 2015, 51, p 75-88

56. F.D. Murnaghan, The Compressibility of Media Under Extreme Pressures, Proc. Natl. Acad. Sci. USA, 1944, 30, p 244-247 